\title{
The impact of body composition parameters on severe toxicities in patients with locoregionally advanced nasopharyngeal carcinoma undergoing neoadjuvant chemotherapy
}

\author{
Xing Xing ${ }^{1,2,3 \#}$, Xin Zhou ${ }^{1,2,3 \#}$, Youqi Yang ${ }^{1,2,3}$, Yujiao $\mathrm{Li}^{1,2,3}$, Chaosu Hu ${ }^{1,2,3}$, Chunying Shen ${ }^{1,2,3}$ \\ ${ }^{1}$ Department of Radiation Oncology, Fudan University Shanghai Cancer Center, Shanghai, China; ${ }^{2}$ Department of Oncology, Shanghai Medical \\ College, Fudan University, Shanghai, China; ${ }^{3}$ Shanghai Key Laboratory of Radiation Oncology, Shanghai, China \\ Contributions: (I) Conception and design: C Hu, C Shen; (II) Administrative support: C Hu; (III) Provision of study materials or patients: C Hu, C \\ Shen; (IV) Collection and assembly of data: X Zhou, Y Yang; (V) Data analysis and interpretation: X Xing, X Zhou; (VI) Manuscript writing: All \\ authors; (VII) Final approval of manuscript: All authors. \\ \#These authors contributed equally to this work. \\ Correspondence to: Prof. Chaosu Hu; Prof. Chunying Shen. Address: Fudan University Shanghai Cancer Center, 270 Dongan Road, Shanghai 200032 , \\ China. Email: hucsu62@163.com; shency2016@163.com.
}

\begin{abstract}
Background: Neoadjuvant chemotherapy (NACT) treatment in locoregionally advanced nasopharyngeal carcinoma (LA-NPC) can lead to considerable toxicity. Loss of skeletal muscle mass showed relevance with increased chemotherapy-related toxicity and poor survival in various cancer types, but its significance in NPC remains unclear. This study aimed to investigate the relationship between body composition parameters and the incidence of NACT toxicity in LA-NPC patients.

Methods: Ninety-six LA-NPC patients were retrospectively enrolled. All patients had pre-treatment abdominal computed tomography (CT) images to exclude distant metastasis. Lean body mass (LBM, $\mathrm{kg}$ ) was estimated based on cross-sectional muscle area at the third lumbar vertebra (L3) level on CT, and skeletal muscle index (SMI, $\mathrm{cm}^{2} / \mathrm{m}^{2}$ ) was calculated. Doses of chemotherapeutics were normalized as dose/LBM $(\mathrm{mg} / \mathrm{kg})$. Grade 3-4 toxicity was defined as severe. The associations between body composition parameters and severe toxicities were assessed using univariate and multivariate logistic regression analyses. Optimal cutoff points were obtained with a receiver operating characteristic (ROC) curve.

Results: Of the 96 patients, $81.2 \%$ received the docetaxel + cisplatin (TP) regimen, and the rest received the gemcitabine + cisplatin (GP) regimen. Males had more LBM and a higher SMI at baseline, and females received a markedly higher dose of docetaxel and gemcitabine per $\mathrm{kg} \operatorname{LBM}(\mathrm{P}<0.001)$. With a cutoff value of $52.7 \mathrm{~cm}^{2} / \mathrm{m}^{2}$, patients with higher SMI showed lower risk of severe toxicity. For TP regimen group, those presented with grade 3-4 neutropenia had a higher dose per $\mathrm{kg}$ LBM. Univariate and multivariate analyses showed that the LBM-adjusted dose was significantly associated with severe neutropenia in the TP regimen group $(\mathrm{P}<0.001)$. The LBM-normalized docetaxel cutoff value of $2.64 \mathrm{mg} / \mathrm{kg}$ was a prominent predictor of $\geq$ grade 3 neutropenia $(\mathrm{P}=0.003)$, but a higher dose of docetaxel per $\mathrm{kg}$ LBM did not provide a better objective response rate.

Conclusions: LA-NPC patients with lower SMI and higher dose of docetaxel per $\mathrm{kg}$ LBM are more likely to suffer from severe treatment-related toxicity. Higher docetaxel dose per $\mathrm{kg}$ LBM is a prominent predictor for severe neutropenia, but not for NACT response. LBM showed good potential in toxicity risk prediction and dose determination.
\end{abstract}

Keywords: Locoregionally advanced nasopharyngeal carcinoma (LA-NPC); neoadjuvant chemotherapy (NACT); body composition; lean body mass (LBM); skeletal muscle index (SMI)

Submitted Jun 04, 2021. Accepted for publication Jul 15, 2021.

doi: $10.21037 /$ atm-21-3412

View this article at: https://dx.doi.org/10.21037/atm-21-3412 


\section{Introduction}

For locoregionally advanced nasopharyngeal carcinoma (LA-NPC), neoadjuvant chemotherapy (NACT) prior to concurrent chemotherapy (CCRT) has been reported to improve failure-free survival compared with CCRT alone, and platinum-based NACT regimens have been established as standard treatment options for LA-NPC $(1,2)$. Consistent with most other chemotherapy regimens, the NACT dosage for NPC patients has long been determined according to patients' body surface area (BSA) in routine clinical practice. However, it is evident in clinical practice that chemotherapy toxicity varies among patients with identical BSA. The role of BSA in the calculation of chemotherapy dose has been questioned.

Interest has been drawn to the impact of body composition and lean body mass (LBM). In various cancer types, the depletion of LBM is associated with decreased survival and an increased risk of complications and systemic treatment-related toxicity (3-6), and has been proposed as a predictor of treatment tolerance, toxicity, and outcome. LBM can be conveniently estimated from a single abdominal cross-sectional image on computed tomography (CT) scans that are routinely acquired for pre-treatment staging and treatment evaluation, and can be quantified by the skeletal muscle index (SMI). However, the relationship between body composition and the toxicity of NACT has never been studied for LA-NPC patients. The aim of this study is to investigate the impact and predictive value of body composition on chemotherapy toxicity in LA-NPC patients treated with NACT. We present the following article in accordance with the STARD reporting checklist (available at https://dx.doi.org/10.21037/atm-21-3412).

\section{Methods}

\section{Cobort population}

Patients who were newly diagnosed as stage II-IVa NPC $\left(7^{\text {th }}\right.$ edition of the American Joint Committee on Cancer and the International Union for Cancer Control staging system) between January 2018 and April 2019 in our hospital were consecutively enrolled into the retrospective study. The inclusion criteria were as follows: (I) histopathology confirmed NPC; (II) had a pre-treatment abdominal CT scan or PET/CT scan within 30 days before treatment; (III) received NACT with the standard TP regimen (docetaxel $75 \mathrm{mg} / \mathrm{m}^{2} \mathrm{~d} 1+$ cisplatin $\left.25 \mathrm{mg} / \mathrm{m}^{2} \mathrm{~d} 1-3\right)$ or GP regimen (gemcitabine $1 \mathrm{~g} / \mathrm{m}^{2} \mathrm{~d} 1,8+$ cisplatin $25 \mathrm{mg} / \mathrm{m}^{2} \mathrm{~d} 1-3$ ) according to our center's routine; (IV) response to treatment was evaluated by MRI after NACT. BSA was calculated using the Mosteller formula $\left\{\right.$ BSA $\left(\mathrm{m}^{2}\right)=[$ height $(\mathrm{cm}) \times$ weight $\left.(\mathrm{kg})] / 3,600\}^{1 / 2}\right\}$. Clinical profiles including body weight, height, Karnofsky performance score (KPS), and calculated BMI were collected before treatment. Toxicities were graded according to the Common Terminology Criteria for Adverse Events Version 4.03 (CTCAE 4.03), and toxicity profiles during the $1^{\text {st }}$ cycle of NACT were collected. Grades 3-4 were defined as severe toxicity. This study was conducted in accordance with the Declaration of Helsinki (as revised in 2013), and was approved by the Fudan University Shanghai Cancer Center Ethics Committee (No. 1505146-2). Informed consent was waived because of the retrospective nature of the study, analysis was conducted on anonymous clinical data.

\section{Body composition assessment}

The abdominal CT data was collected, either from abdominal CT or PET/CT scans. Skeletal muscle tissue was identified on the axial image of the third lumbar vertebra (L3) by Hounsfield unit (HU) range settings from -29 to $+150 \mathrm{HU}$, and the range of skeletal muscle was delineated manually. Cross-sectional muscle area (CSA) referred to the sum of the delineated areas. All CT images were analyzed by a single researcher, who was blinded of patients' toxicity and induction chemotherapy response. LBM $(\mathrm{kg})$ was estimated by the regression equation: $\mathrm{LBM}(\mathrm{kg})=0.30 \times \mathrm{L} 3$ muscle CSA $\left(\mathrm{cm}^{2}\right)+6.06$, which was reported in previous research by Mourtzakis et al. (7). The SMI $\left(\mathrm{cm}^{2} / \mathrm{m}^{2}\right)$ was defined as CSA $\left(\mathrm{cm}^{2}\right)$ at the L3 level on CT divided by height squared $\left(\mathrm{m}^{2}\right)$. The doses of docetaxel and gemcitabine were normalized by $\mathrm{LBM}$ as dose/LBM $(\mathrm{mg} / \mathrm{kg})$. The criteria for the diagnosis of sarcopenia reported by earlier studies by Mourtzakis et al. (7) and Martin et al. (8) were applied for the included patients to compare the difference between defined sarcopenia and nonsarcopenia patients.

\section{Statistical analysis}

Descriptive statistics were used to examine the baseline characteristics of the patients. Continuous data were represented as mean \pm standard deviation (SD). Categorical data were represented as the number and percentage of the total. Comparisons of clinical characteristics and toxicities between two groups were analyzed using Pearson chi-square test or Fisher's exact test, and the independent samples 
Table 1 Patient characteristics

\begin{tabular}{|c|c|}
\hline Characteristic & Number (\%) \\
\hline Age (years) & Median: 46.5 [23-70] \\
\hline \multicolumn{2}{|l|}{ Gender } \\
\hline Male & $72(75.0)$ \\
\hline Female & $24(25.0)$ \\
\hline \multicolumn{2}{|l|}{ T stage } \\
\hline $\mathrm{T} 1$ & $5(5.2)$ \\
\hline $\mathrm{T} 2$ & $20(20.8)$ \\
\hline T3 & $37(38.5)$ \\
\hline $\mathrm{T} 4$ & $34(35.5)$ \\
\hline \multicolumn{2}{|l|}{$\mathrm{N}$ stage } \\
\hline NO & $1(1.0)$ \\
\hline N1 & $21(21.9)$ \\
\hline N2 & $47(49.0)$ \\
\hline N3 & $27(28.1)$ \\
\hline \multicolumn{2}{|l|}{ Clinical stage } \\
\hline II & $1(1.0)$ \\
\hline III & $40(41.7)$ \\
\hline IV & $55(57.3)$ \\
\hline \multicolumn{2}{|l|}{ KPS (\%) } \\
\hline 100 & $85(88.5)$ \\
\hline 90 & $10(10.4)$ \\
\hline 80 & $1(1.1)$ \\
\hline \multicolumn{2}{|c|}{ Chemotherapy regimen } \\
\hline TP & 78 (81.3) \\
\hline GP & $18(18.7)$ \\
\hline \multicolumn{2}{|l|}{ Cycles of NACT } \\
\hline 1 & $2(2.1)$ \\
\hline 2 & $79(82.3)$ \\
\hline 3 & $15(15.6)$ \\
\hline \multicolumn{2}{|l|}{ BMI $\left(\mathrm{kg} / \mathrm{m}^{2}\right)$} \\
\hline$\geq 25$ & $36(37.5)$ \\
\hline$<25$ & $60(62.5)$ \\
\hline Total & 96 \\
\hline
\end{tabular}

KPS, Karnofsky performance score; NACT, neoadjuvant chemotherapy; BMI, body mass index. $t$-test. Logistic regression was used to test the association between clinical characteristics and the incidence of severe toxicity. For multivariate logistic regression, the forward stepwise (likelihood ratio) method of entering the model was selected. The optimal cutoff points for SMI- and LBMnormalized chemotherapy dose were determined by a receiver operating characteristic (ROC) curve. A P value $<0.05$ was considered significant for all tests. All statistical analyses were performed using SPSS version 25 (Armonk, NY: IBM Corp.).

\section{Results}

\section{Patient characteristics}

A total of 96 consecutive patients were enrolled. Of these patients, $75 \%$ were male, with a median age of 46.5 years (range, $23-70$ years). Seventy-eight patients $(81.3 \%$ ) received the TP regimen, while the other 18 patients $(18.8 \%)$ received the GP regimen. Two cycles of NACT were applied in 79 patients. Two patients received only 1 cycle of NACT as patients refused the second cycle due to intolerance, although no grade 3-4 toxicity was observed after the $1^{\text {st }}$ cycle. Additionally, 15 patients received 3 cycles with good tolerance and response to the regimen. The patient characteristics are listed in Table 1.

\section{Toxicity}

Grade 3-4 hematological toxicity was observed in 52 patients $(54.2 \%)$. The predominant hematological toxicities were neutropenia for TP and thrombocytopenia for GP. Twenty-one patients presented with elevated serum alanine aminotransferase (ALT) indicating liver dysfunction after the $1^{\text {st }}$ cycle. Among these patients, only 1 who received the TP regimen reached grade 3 . Thirteen patients presented with increased creatinine, none of which were over grade 1 . The incidence of NACT-related toxicity is summarized in Table 2.

\section{Differences in body composition and toxicity stratified by gender}

At baseline, males had significantly higher LBM (mean: 52.6 vs. $37.1 \mathrm{~kg}, \mathrm{P}<0.001$ ) and SMI (mean: 53.4 vs. $41.1 \mathrm{~cm}^{2} / \mathrm{m}^{2}$, $\mathrm{P}<0.001$, Figure 1) than females before treatment. The 
Table 2 Incidence of NACT-related grade 3-4 toxicity

\begin{tabular}{lccc}
\hline NACT-related toxicity & GP $(\mathrm{N}=18), \mathrm{n}(\%)$ & $\mathrm{TP}(\mathrm{N}=78), \mathrm{n}(\%)$ & $\mathrm{P}$ value \\
\hline Total toxicity & $11(61.1)$ & $41(52.6)$ & 0.512 \\
Neutropenia & $7(38.9)$ & $39(50.0)$ & 0.395 \\
Thrombocytopenia & $7(38.9)$ & $0(0)$ & $<0.001$ \\
Acute liver injury & 0 & $1(1.3)$ & 0.812 \\
Acute kidney injury & 0 & 0 & - \\
\hline
\end{tabular}

Italic $\mathrm{P}$ value indicates statistically significant difference. NACT, neoadjuvant chemotherapy.

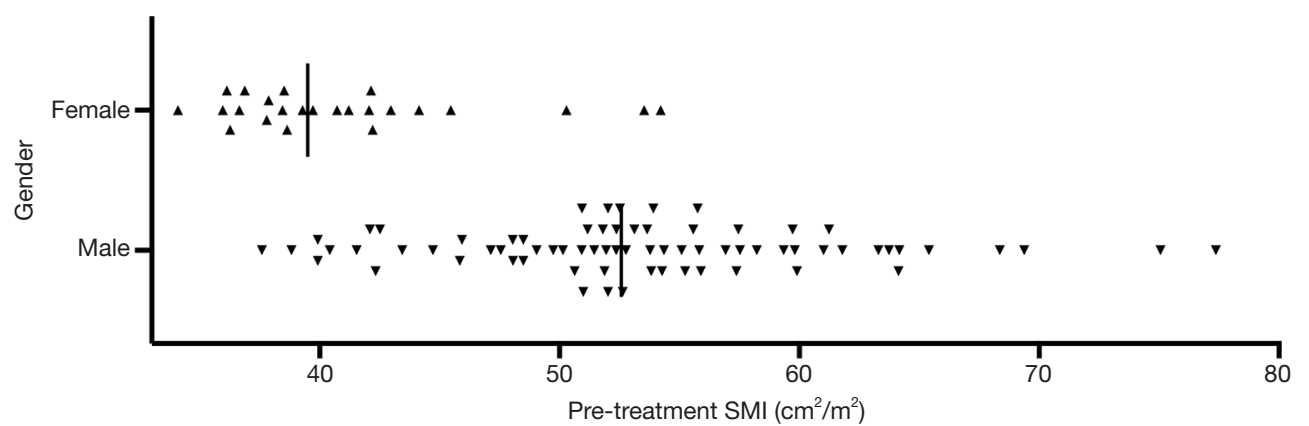

Figure 1 Distribution of SMI in female and male patients (median number of each group marked in vertical line). SMI, skeletal muscle index.

Table 3 Comparison of body composition, chemotherapy dose and toxicity between male and female patients

\begin{tabular}{lccc}
\hline Parameter & Male $(\mathrm{n}=72)$ & Female $(\mathrm{n}=24)$ & P value \\
\hline Pretreatment SMI $\left(\mathrm{cm}^{2} / \mathrm{m}^{2}\right.$, mean \pm SD) & $53.4 \pm 8.1$ & $41.1 \pm 5.3$ & $<0.001$ \\
Pretreatment LBM $(\mathrm{mg} / \mathrm{kg}$, mean $\pm \mathrm{SD})$ & $52.6 \pm 7.1$ & $37.2 \pm 3.6$ & 6.001 \\
$\geq$ grade 3 overall toxicity (\%) & 50.0 & $16(66.7)$ & 0.156 \\
$\geq$ grade 3 hematological toxicity (\%) & $35(48.6)$ & 66.7 & 0.125 \\
$\geq$ grade 3 neutropenia (\%) & 41.7 & 8.3 & 0.034 \\
$\geq$ grade 3 thrombocytopenia (\%) & 6.9 & 3.08 & 0.562 \\
LBM-adjusted Docetaxel $(\mathrm{mg} / \mathrm{kg})$ & 2.49 & 79.44 & $<0.001$ \\
LBM-adjusted Gemcitabine $(\mathrm{mg} / \mathrm{kg})$ & 62.73 & $<0.001$ & \\
\hline
\end{tabular}

Italic $\mathrm{P}$ values indicate statistically significant difference. SMI, skeletal muscle index; LBM, lean body mass.

incidence of toxicities also differed between genders. A higher rate of NACT-related toxicity was observed in females, although the differences in overall toxicity and hematological toxicity only approached statistical significance $(\mathrm{P}=0.156$ and 0.125 , respectively). The incidence of grade $3-4$ neutropenia differed significantly between the two genders $(66.7 \% v s$. $41.7 \%, \mathrm{P}=0.034$ ) (Table 3). When the dose of chemotherapy was normalized by LBM, females received markedly higher doses of docetaxel (mean: 3.08 vs. $2.49 \mathrm{mg} / \mathrm{kg}, \mathrm{P}<0.001$ ) and gemcitabine (mean: 79.4 vs. $62.7 \mathrm{mg} / \mathrm{kg}, \mathrm{P}<0.001$ ).

\section{Cutoff value of SMI as a predictor of NACT-related toxicity}

Using the diagnostic criteria reported by Mourtzakis et al. (7), which was SMI $<52.4 \mathrm{~cm}^{2} / \mathrm{m}^{2}$ for men and $<38.5 \mathrm{~cm}^{2} / \mathrm{m}^{2}$ for women, 33 patients met the diagnosis of sarcopenia, but the incidence of grade 3-4 toxicity was close between 


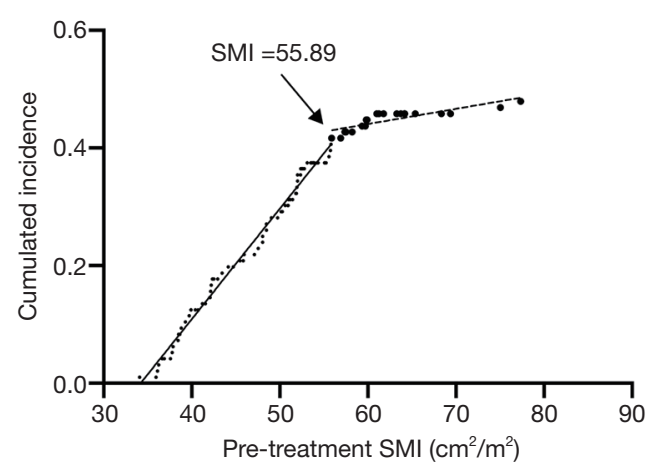

Figure 2 Cumulated incidence of severe NACT-related toxicity with SMI. NACT, neoadjuvant chemotherapy; SMI, skeletal muscle index.

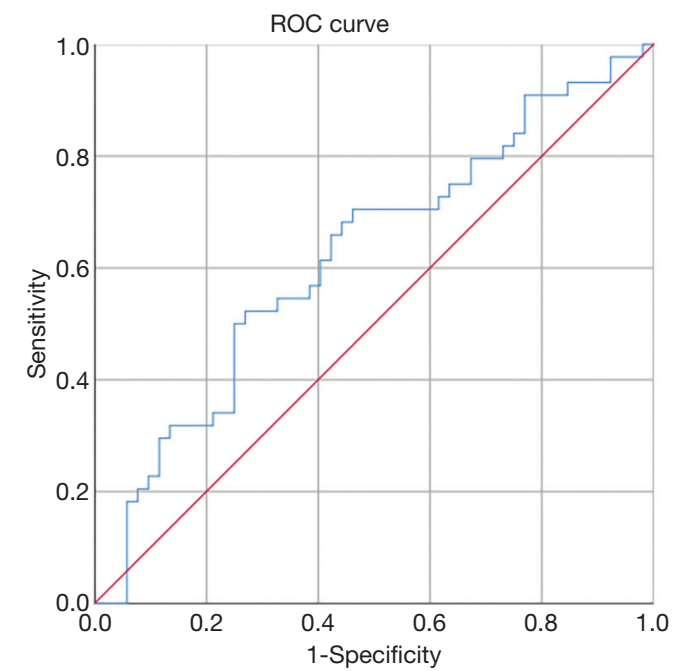

Figure 3 ROC curve analysis by grade 3-4 overall toxicity to determine optimal cutoff value for SMI $\left(52.7 \mathrm{~cm}^{2} / \mathrm{m}^{2}\right)$. Area under the ROC curve of sarcopenia was 0.621 (95\% confidence interval: 0.508-0.734; $\mathrm{P}=0.019)$. ROC, receiver operating characteristic; SMI, skeletal muscle index.

the two groups $(57.6 \%$ vs. $52.4 \%, \mathrm{P}=0.628)$. The criteria reported by Martin et al. (SMI $<43 \mathrm{~cm}^{2} / \mathrm{m}^{2}$ for males if BMI $<24.9 \mathrm{~kg} / \mathrm{m}^{2}$ or SMI $<53 \mathrm{~cm}^{2} / \mathrm{m}^{2}$ for males if $\mathrm{BMI}>25 \mathrm{~kg} / \mathrm{m}^{2}$, and SMI $<41 \mathrm{~cm}^{2} / \mathrm{m}^{2}$ for females) also showed no efficacy in risk stratification between the two groups $(60.5 \%$ and $49.1 \%$, $\mathrm{P}=0.265)$. The cumulative incidence of overall toxicity was plotted in Figure 2, which indicated that when the SMI was below the inflection point of $55.89 \mathrm{~cm}^{2} / \mathrm{m}^{2}$, the cumulative incidence grew steadily and rapidly as the SMI increased, and when the SMI was over $55.89 \mathrm{~cm}^{2} / \mathrm{m}^{2}$, the incidence of toxicity

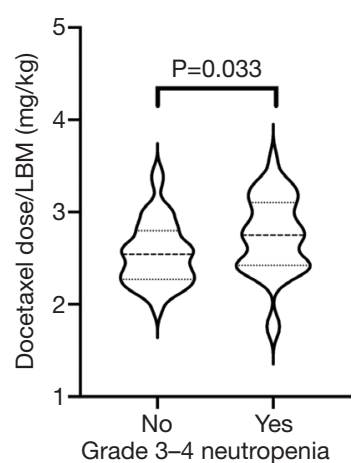

Figure 4 Distribution of docetaxel dose per LBM in patients with or without grade 3-4 neutropenia. LBM, lean body mass.

fell abruptly. An ROC curve was further utilized to analyze the relationship between toxicity and body compositions. We identified the optimal cutoff value of the SMI at $52.7 \mathrm{~cm}^{2} / \mathrm{m}^{2}$ (95\% confidence interval: 0.508-0.734; $\mathrm{P}=0.019)$. Patients with a lower SMI suffered more frequent toxicities (64.4\% vs. $37.8 \%, \mathrm{P}=0.019$ ) (Figure 3).

\section{Relationship between LBM-normalized docetaxel dose and severe neutropenia}

Since the number of patients in the GP group was limited and the two regimens showed different toxicities, further regression analysis of the relationship between body composition and toxicity was performed within the TP regimen group. As shown in Figure 4, the average dose of docetaxel per $\mathrm{kg}$ LBM was higher in patients with grade 3-4 neutropenia than in those without $(\mathrm{P}=0.033$, Figure 4). In the univariate logistic regression, gender, pre-treatment SMI, and dose of docetaxel per $\mathrm{kg}$ LBM showed a possible relationship with the incidence of grade 3-4 neutropenia. Age and KPS, which are generally accepted to influence treatment tolerance, were also included into the multivariate regression. The results showed that LBM-adjusted dose was the only factor that was significantly associated with severe neutropenia in the TP regimen group $(\mathrm{P}<0.001)$ (Table 4). An ROC curve was generated for docetaxel dose/ LBM using grade 3-4 neutropenia as an end point, with a cutoff value of $2.64 \mathrm{mg} / \mathrm{kg}$ (area under the curve: 0.654 , $95 \%$ confidence interval: $0.532-0.776, \mathrm{P}=0.019)$. Patients who received a higher docetaxel dose per $\mathrm{kg}$ LBM had a much higher frequency of $\geq$ grade 3 neutropenia $(65.7 \%$ vs. $37.2 \%, \mathrm{P}=0.012$ ) (Figure 5). However, after the end of induction chemotherapy, the response rate of the high dose 
Table 4 Logistic regression analysis of grade 3-4 neutropenia for TP regimen group

\begin{tabular}{|c|c|c|c|}
\hline Characteristic & \multicolumn{2}{|c|}{ Univariate } & $\frac{\text { Multivariate }}{\mathrm{P} \text { value }}$ \\
\hline Gender & $0.037(1.062-7.382)$ & 0.037 & 0.367 \\
\hline Age & $1.010(0.970-1.051)$ & 0.641 & 0.507 \\
\hline KPS & $0.992(0.888-1.108)$ & 0.887 & 0.702 \\
\hline Pretreatment SMI & $0.947(0.903-0.993)$ & 0.025 & - \\
\hline Dose of docetaxel & $0.968(0.923-1.014)$ & 0.168 & - \\
\hline Dose of docetaxel/LBM & $3.733(1.078-12.963)$ & 0.038 & 0.038 \\
\hline
\end{tabular}

Italic $\mathrm{P}$ values indicate statistically significant difference. KPS, Karnofsky performance score; SMI, skeletal muscle index; LBM, lean body mass.

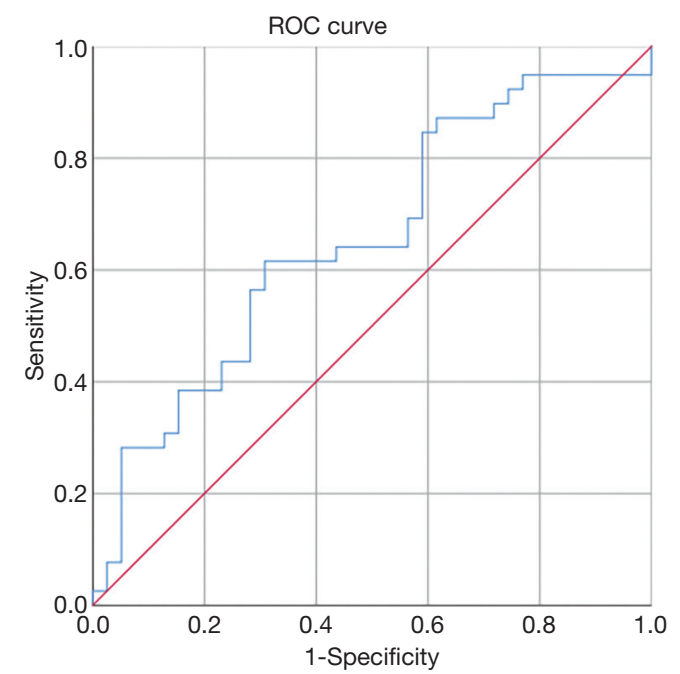

Figure 5 ROC curve analysis by grade 3-4 neutropenia to determine optimal cutoff value for LBM-adjusted Docetaxel $(2.64 \mathrm{mg} / \mathrm{kg})$ in TP subgroup. Area under the ROC curve of sarcopenia was 0.654 (95\% confidence interval: 0.532-0.776; $\mathrm{P}=0.019)$. ROC, receiver operating characteristic; LBM, lean body mass.

group was $80 \%$, which did not differ from that of the low dose group (72.2\%, $\mathrm{P}=0.439$, Figure 6).

\section{Discussion}

The results of the current study showed that the incidence of NACT-related toxicity differed between males and females, which could be explained by differences in body composition represented by the SMI and LBM. Given

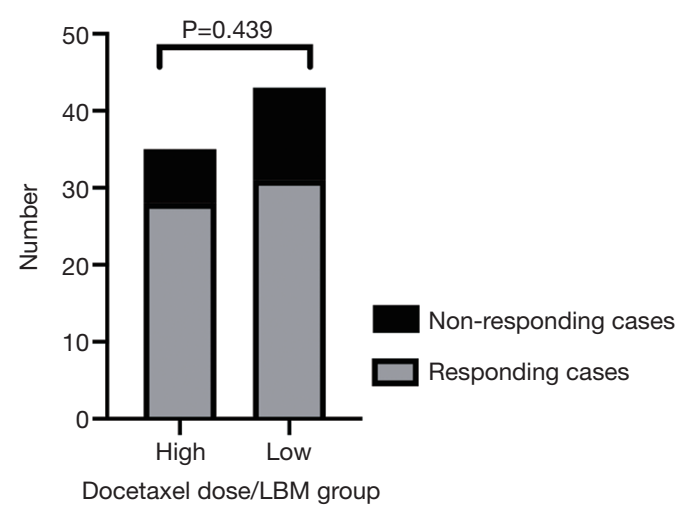

Figure 6 Response rate in docetaxel dose per LBM groups divided by ROC curve-identified cutoff value. ROC, receiver operating characteristic; LBM, lean body mass.

the standardized dose of NACT calculated based on BSA, patients with lower LBM tend to present with a higher rate of toxicity. A stronger relationship was observed between the chemotherapeutic dose normalized by LBM and the incidence of severe toxicity for LA-NPC patients. These results are consistent with findings in other cancer types. The role of body composition and the nutritional status of cancer patients have garnered much attention over the past decades. It has already been reported in many cancers that sarcopenia or lower LBM is related to higher rates of severe toxicities (9), more treatment complications (10), and worse clinical outcomes $(11,12)$ in multiple treatment modalities. A recent retrospective study based on a large cohort of NPC patients showed that sarcopenia was an independent predictor of overall survival and distant metastasis free survival, and higher rates of toxicities during radiotherapy 
were observed in patients with sarcopenia (13). However, there is a lack of research on the toxicity of NACT. NACT has been proven to confer a progression-free survival advantage over CCRT alone and can reduce the tumor burden for a smaller target volume in radiotherapy when critical structures are abutting or invaded $(1,2)$. However, NACT regimens for LA-NPC usually include cisplatin plus docetaxel or gemcitabine, bringing about not only better survival, but also a considerable rate of severe toxicity, which may influence patients' performance statuses and cause delays in definitive chemoradiotherapy. Therefore, it is crucial to take careful consideration of the personalized dosing of NACT to maximize the therapeutic benefit and reduce harm.

BSA-based dosing strategies for most antineoplastic agents initiated since the 1950 s originally aimed to minimize interindividual variation, and have long been established as standards for the dosing of most anticancer agents $(14,15)$. However, clinical practice has proven their limited ability to balance variation within the population of cancer patients (16), as in the current study, the BSA-based dosing strategy failed to equalize the distribution of toxicities between the two genders. Therefore, attention has been paid to other anthropometric parameters such as LBM.

The relationship between LBM and dosing should be considered from multiple aspects. Body composition is made up of fat mass and LBM, and muscle is considered as a diffusion compartment for cancer therapeutics (17). On the one hand, it is speculated that with more skeletal muscle, the concentrations of anticancer agents may be lowered in circulation, thus reducing the incidence of severe chemotherapy toxicity, especially hematological toxicity (18). On the other hand, it was also proposed that muscle mass gain increases chemotherapeutic clearance, which may be associated with decreased efficacy (19). In the current study, the incidence of severe toxicity dropped when the SMI was over $55.89 \mathrm{~cm}^{2} / \mathrm{m}^{2}$. However, it made no difference to the response rates between the higher and lower LBMnormalized dose groups. Therefore, factoring LBM into the calculation of chemotherapy dose may help to optimize the personalization of chemotherapy for NPC patients receiving NACT. Whether there are possible benefits of dose escalation for the higher SMI population to gain a better response, and whether there is a benefit of dose deescalation for those with a lower SMI to reduce toxicity, will be further explored in future studies.

CT has been utilized as an effective way of measuring human body composition. Measurement of a single abdominal image can provide estimates of total body skeletal muscle and adipose tissue. The relationship between muscle area on L3 level CT images and total body mass has been recognized, and body composition estimation and SMI calculation based on L3 axial images has been established as the standard method and is widely accepted (20,21). In our study, this verified method was also adopted. Based on this technique for body composition measurement, previous publications have proposed several different definitions of low skeletal muscle mass/sarcopenia, among which the Mourtzakis et al. (7) and Martin et al. (8) criteria based on large populations of respiratory and gastrointestinal cancer patients have been mostly employed. In previous studies, the prevalence of sarcopenia differed significantly with different criteria (22), even for the same kinds of malignancies within the same race, and the above two criteria failed to stratify toxicity risk in the LA-NPC population in the cohort of our study. Theoretically, nutritional status and body composition should vary by age, gender, race, and specifically for cancer patients, the primary site and stage. Therefore, there have been several studies that have aimed to define an optimal cutoff value for sarcopenia. In the current study, the SMI cutoff point identified by the ROC curve could predict the risk of NACT-induced severe toxicity, and the cutoff value of the docetaxel dose per $\mathrm{kg}$ LBM also showed efficacy in risk stratification for grade 3-4 neutropenia in the TP regimen group. These cutoff values were specific to the LA-NPC patients included in this study, and need to be further verified by larger populations with the same characteristics.

This is the first study focusing on the influence of body composition on the dosing and toxicity of NACT in LANPC patients. Several limitations should be noted. First, the comparatively small sample size is the main limitation of our study. The retrospective nature of this study also limited the reliability of the cutoff values acquired. This research is a preliminary exploration into the relationship between body composition and chemotherapy toxicity and outcome in NPC patients. Verification of the relationship based on larger prospective cohort should be performed in order to make LBM more easily and reliably incorporated into the dose calculation of NACT for LA-NPC patients. Second, as the follow-up period of the cohort was short, and data on survival was lacking, the prognostic value of body composition is to be further studied. Comprehensive analysis which takes into account not only toxicity but also survival might have more profound significance and could help optimize our clinical routine of chemotherapy dosage. 
Based on the enhanced understanding of the effects of body composition on the clinical outcome of LA-NPC patients, it is worth further investigating whether timely nutritional interventions may help to maintain muscle mass and lower the rate of severe toxicity, thus leading to better tolerance and improved prognosis.

\section{Conclusions}

LA-NPC patients with low SMI are more likely to experience severe treatment-related toxicity during NACT. A higher dose of docetaxel per $\mathrm{kg} L B M$ is related to an increased incidence of toxicity, yet does not contribute to a better response rate. While BSA was insufficient in predicting toxicity, LBM showed good potential in guiding the NACT dose for NPC patients. Future efforts are warranted to optimize the dosing strategy to reduce toxicity for patients with a lower SMI, and to increase the response rate for patients with a higher SMI to further improve prognosis.

\section{Acknowledgments}

Funding: This study was funded by National Key Technologies Research and Development Program on Prevention and Control of Chronic Non-communicable Diseases (Grant No. 2018YFC1313204).

\section{Footnote}

Reporting Checklist: The authors have completed the STARD reporting checklist. Available at https://dx.doi. org/10.21037/atm-21-3412

Data Sharing Statement: Available at https://dx.doi. org/10.21037/atm-21-3412

Conflicts of Interest: All authors have completed the ICMJE uniform disclosure form (available at https://dx.doi. org/10.21037/atm-21-3412). The authors have no conflicts of interest to declare.

Ethical Statement: The authors are accountable for all aspects of the work in ensuring that questions related to the accuracy or integrity of any part of the work are appropriately investigated and resolved. All procedures performed in studies involving human participants were approved by Fudan University Shanghai Cancer Center Ethics Committee
(No. 1505146-2) and in accordance with the Declaration of Helsinki (as revised in 2013). Informed consent was waived because of the retrospective nature of the study, analysis was conducted on anonymous clinical data.

Open Access Statement: This is an Open Access article distributed in accordance with the Creative Commons Attribution-NonCommercial-NoDerivs 4.0 International License (CC BY-NC-ND 4.0), which permits the noncommercial replication and distribution of the article with the strict proviso that no changes or edits are made and the original work is properly cited (including links to both the formal publication through the relevant DOI and the license). See: https://creativecommons.org/licenses/by-nc-nd/4.0/.

\section{References}

1. Sun Y, Li WF, Chen NY, et al. Induction chemotherapy plus concurrent chemoradiotherapy versus concurrent chemoradiotherapy alone in locoregionally advanced nasopharyngeal carcinoma: a phase 3 , multicentre, randomised controlled trial. Lancet Oncol 2016;17:1509-20.

2. Ni W, Qi W, Xu F, et al. Prognostic value of nasopharynx tumour volume in local-regional advanced nasopharyngeal carcinoma. Ann Transl Med 2020;8:223.

3. Blauwhoff-Buskermolen S, Versteeg KS, de van der Schueren MA, et al. Loss of Muscle Mass During Chemotherapy Is Predictive for Poor Survival of Patients With Metastatic Colorectal Cancer. J Clin Oncol 2016;34:1339-44.

4. Kim EY, Kim YS, Park I, et al. Prognostic Significance of CT-Determined Sarcopenia in Patients with Small-Cell Lung Cancer. J Thorac Oncol 2015;10:1795-9.

5. Panje CM, Höng L, Hayoz S, et al. Skeletal muscle mass correlates with increased toxicity during neoadjuvant radiochemotherapy in locally advanced esophageal cancer: A SAKK 75/08 substudy. Radiat Oncol 2019;14:166.

6. Qiang JK, Lipscombe LL, Lega IC. Association between diabetes, obesity, aging, and cancer: review of recent literature. Transl Cancer Res 2020;9:5743-59.

7. Mourtzakis M, Prado CM, Lieffers JR, et al. A practical and precise approach to quantification of body composition in cancer patients using computed tomography images acquired during routine care. Appl Physiol Nutr Metab 2008;33:997-1006.

8. Martin L, Birdsell L, Macdonald N, et al. Cancer cachexia in the age of obesity: skeletal muscle depletion is a powerful prognostic factor, independent of body mass 
index. J Clin Oncol 2013;31:1539-47.

9. Palmela C, Velho S, Agostinho L, et al. Body composition as a prognostic factor of neoadjuvant chemotherapy toxicity and outcome in patients with locally advanced gastric cancer. J Gastric Cancer 2017;17:74-87.

10. Ansari E, Chargi N, van Gemert JTM, et al. Low skeletal muscle mass is a strong predictive factor for surgical complications and a prognostic factor in oral cancer patients undergoing mandibular reconstruction with a free fibula flap. Oral Oncol 2020;101:104530.

11. van Rijn-Dekker MI, van den Bosch L, van den Hoek JGM, et al. Impact of sarcopenia on survival and late toxicity in head and neck cancer patients treated with radiotherapy. Radiother Oncol 2020;147:103-10.

12. Zhuang CL, Huang DD, Pang WY, et al. Sarcopenia is an Independent Predictor of Severe Postoperative Complications and Long-Term Survival After Radical Gastrectomy for Gastric Cancer: Analysis from a LargeScale Cohort. Medicine (Baltimore) 2016;95:e3164.

13. Hua X, Liao JF, Huang X, et al. Sarcopenia is associated with higher toxicity and poor prognosis of nasopharyngeal carcinoma. Ther Adv Med Oncol 2020;12:1758835920947612.

14. Pinkel D. The use of body surface area as a criterion of drug dosage in cancer chemotherapy. Cancer Res 1958;18:853-6.

15. Baker RJ, Kozoll DD, Meyer KA, et al. The use of surface area as a basis for establishing normal blood volume. Surg

Cite this article as: Xing X, Zhou X, Yang Y, Li Y, Hu C, Shen C. The impact of body composition parameters on severe toxicities in patients with locoregionally advanced nasopharyngeal carcinoma undergoing neoadjuvant chemotherapy. Ann Transl Med 2021;9(14):1180. doi: 10.21037/atm-21-3412
Gynecol Obstet 1957;104:183-9.

16. Felici A, Verweij J, Sparreboom A, et al. Dosing strategies for anticancer drugs: the good, the bad and body-surface area. Eur J Cancer 2002;38:1677-84.

17. Roubenoff R, Kehayias JJ. The meaning and measurement of lean body mass. Nutr Rev 1991;49:163-75.

18. Hilmi M, Jouinot A, Burns R, et al. Body composition and sarcopenia: The next-generation of personalized oncology and pharmacology? Pharmacol Ther 2019;196:135-59.

19. Chu MP, Lieffers J, Ghosh S, et al. Skeletal muscle density is an independent predictor of diffuse large B-cell lymphoma outcomes treated with rituximab-based chemoimmunotherapy. J Cachexia Sarcopenia Muscle 2017;8:298-304.

20. Shen W, Punyanitya M, Wang Z, et al. Total body skeletal muscle and adipose tissue volumes: estimation from a single abdominal cross-sectional image. J Appl Physiol (1985) 2004;97:2333-8.

21. Derstine BA, Holcombe SA, Ross BE, et al. Skeletal muscle cutoff values for sarcopenia diagnosis using T10 to L5 measurements in a healthy US population. Sci Rep 2018;8:11369.

22. Walowski CO, Braun W, Maisch MJ, et al. Reference Values for Skeletal Muscle Mass - Current Concepts and Methodological Considerations. Nutrients 2020;12:755.

(English Language Editor: C. Beltzar) 